Internationalisation et performance : une approche matricielle appliquée au secteur des équipements électriques

Serge Besanger*, Fabrice Roth**

*Professeur associé - ESCE

sbesanger@gmail.com

$+33638368238$

**Professeur des universités

IAE Lyon - Centre de recherche Magellan - Université Jean

Moulin Lyon 3

Roth.fabrice@gmail.com

+33661905 708 


\title{
Internationalisation et performance : une approche matricielle appliquée au secteur des équipements électriques
}

Résumé : Depuis plus de quarante ans, les chercheurs ont étudié la relation entre l'internationalisation des entreprises et leur performance financière. Alors que la plupart des études révèlent l'existence d'une relation, les chercheurs sont en désaccord sur la linéarité et la forme de la courbe statistique. Dans cet article, la relation est étudiée en utilisant une approche matricielle, avec une application statistique au secteur des équipements électriques. Les résultats montrent la non-linéarité du lien entre performance financière (ROE) et chiffre d'affaires à l'international.

Mots clés : Internationalisation, Performance financière, Matrices, Stratégie, Equipements électriques

\section{International orientation and financial performance: the case of the electrical equipment sector}

\begin{abstract}
For over forty years, researchers have investigated the relationship between the internationalization of firms and their financial performance. While most studies point to the existence of a relationship, researchers disagree on the linearity, if any, and shape of the statistical curve.In this article, the relationship is examined using a matrix approach, with a statistical application to the electrical equipment sector. The results show the nonlinearity of the relationship between financial performance (ROE) and international sales.
\end{abstract}

Key words: Internationalization, Financial performance, Matrices, Strategy, Electricals sector.

\section{Orientación internacional y rendimiento financiero: el caso del sector del material eléctrico}

Resumen : Pormás de cuarentaaños, los investigadores han estudiado la relación entre la internacionalización de las empresas y el desempeñofinanciero. Aunque la mayoría de los estudiosmuestran la existencia de unarelación, los investigadores no están de acuerdo en la linealidad y la forma de la curvaestadística.En este artículo, se examina la relación mediante unenfoque matricial, con una aplicaciónestadística alsector del material eléctrico. Los resultados muestran lano-linealidadde la relación entreel desempeño financiero(ROE) y las ventas internacionales.

Palabras claves: Internacionalización, rendimiento financiero, Matrices, Estrategia, Equipo eléctrico. 


\section{Introduction}

Le lien entre internationalisation de l'entreprise et performance est un thème classique de la littérature managériale académique, et une préoccupation constante des dirigeants, plus particulièrement aujourd'hui dans un contexte d'économie globalisée. Les professionnels ont en général tendance à postuler un lien positif entre internationalisation et performance. En revanche, les études en gestion montrent une relation plus complexe. Si les avantages liés à l'internationalisation, comme le relais de croissance, l'image de marque, la diversification des risques sont avancés depuis longtemps (Reid, 1983 ; Hsu et al., 2003 ; Thomas et al., 2004 ; Contractor, 2007 ; Prange et al., 2010), d'autres travaux soulignent des inconvénients comme l'insuffisante maîtrise des synergies opérationnelles, l'inadéquation de la structure et/ou du management, les risques de gouvernance et institutionnels ou les différences culturelles (Michel, 1986; Johanson et al., 1990 ; Gomez-Mejia, 1997 ; Annavarjula, M., 2000 ; Lu et al., 2004 ; Elango et al., 2007 ; Weerawardena, 2007 ; Qian et al., 2008, Tsao et al., 2012). Au final, cette relation s'exprimerait sous la forme d'une courbe en cloche, avec un effet positif de l'internationalisation sur la performance de l'entreprise dans sa première phase (mesurée par le ROE), puis un effet décroissant en cas d'internationalisation trop rapide (Thomas et al., 2004 ; Hitt et al., 2006 ; Hsu et al., 2006), à condition toutefois que l'entreprise se focalise sur un nombre relativement restreint de marchés-cibles lors de la première phase (Rialp, 2005). Selon d'autres chercheurs, la relation s'exprimerait au contraire sous la forme d'une courbe en cloche inversée, avec un effet négatif de l'internationalisation sur la performance de l'entreprise (mesurée par le ROA) dans sa première phase, suivi d'un effet positif (RiahiBelkaoui, 1998).

Cette indétermination (Ruigrok et al., 2004),reliée d'une part à la très grande variété des acteurs, des contextes, des structures, des politiques, etc. qui caractérisent l'activité internationale, d'autre part au rôle de l'aléatoire dans les systèmes globalisés d'une complexité croissante,entretient la nécessité d'améliorer les outils de suivi des activités internationales, en relation avec la performance de l'entreprise. Cette nécessité est plus prégnante encore depuis la dernière crise financière. En effet, des différences notables de croissance économique sont apparues entre pays émergents et développés, rendant l'activité à l'international pour les entreprises occidentales, en particulier françaises, cruciales pour la performance des entreprises. De plus, les politiques monétaires des banques 
centrales (notamment les QE - Quantitative Easing) exercent également une influence sur la croissance économique des Etats. Dans ce contexte, c'est plus précisément la question du relais de croissance qui se trouve posée.Aussi, l'étude spécifique du portefeuille d'activités des entreprises hors de leur marché domestique reste une préoccupation majeure. C'est l'objet de notre article.

Pour mener de telles études, on s'appuiegénéralement en première analyse sur les informations communiquées dans les rapports annuels, à savoir la ventilation du chiffre d'affaires par zones géographiques ${ }^{1}$.Les analyses financières plus poussées vont évidemment tenir compte de l'attractivité de chaque zone pour évaluer le potentiel que représentent les activités à l'international pour une entreprise. Cependant, même si les comparaisons avec les concurrents sont explicitées, ou au moins implicites, on constate l'absence d'outils simples permettant de formaliser : (1) le potentiel du positionnement à l'international des différents acteurs d'un secteur, (2) la dynamique de l'entreprise à l'international.

Pour répondre à cette attente sur le portefeuille des activités à l'international des entreprises, il semble intéressant de recourir à une approche par les matrices qui présente justement l'avantage de pouvoir combiner les deux paramètres clés évoqués plus haut : la part de l'activité à l'international et l'attractivité des zones géographiques. De plus, cette approche, mise dans une perspective historique, permet d'identifier une trajectoire propre à l'entreprise.

C'est donc le principal objectif du présent article de présenter un outil d'analyse du portefeuille d'activités internationales des entreprises sous forme matricielle. De fait, nous attendons que cet outil signale aux investisseurs les dynamiques positives ou négatives des entreprises sur cette dimension.

Nous rappelons dans un premier temps les points clés fondant une approche par les matrices. Le principe de construction d'une matrice du portefeuille international (MPI)est ensuite présenté, avant d'être appliqué à un échantillon d'entreprises du secteur des équipements électriques.

\footnotetext{
${ }^{1}$ Les rapports fournissent également des informations sur la ventilation du chiffre d'affaires par domaine d'activités de l'entreprise.
} 


\section{L'approche par les matrices en management stratégique}

\subsection{Présentation générale}

Les matrices sont des outils particulièrement appréciés en management, et répandus dans les cabinets de conseils. La raison, simple, est liée à leur mode de construction: les deux axes d'une matrice représentent deux paramètres synthétiques permettant d'expliquer une réalité complexe. Cette simplification, qui permet donc d'accompagner les décisions opérationnelles des entreprises, repose en amont sur une identification des variables «explicatives » les plus pertinentes. En ce sens, le principe de construction d'une matrice reflète un mode de pensée inhérent à l'exercice de responsabilités d'ordre stratégique. Par ailleurs, cette simplification apporte une aide utile aux investisseurs quant à la décision d'investissement.

Une matrice présente en effet une double fonction : situer une entité (e.g. une entreprise) au sein d'un groupe de référence (e.g. un secteur d'activité), et déterminer la trajectoire de cette entité par rapport à la trajectoire moyenne du groupe (figure 1).

Aussi, l'axe horizontal correspond généralement à la position de l'entité au sein du groupe et l'axe vertical à son potentiel. La position, forte ou fragile, indique le niveau des ressources mobilisables sur le moment par l'entité ; le potentiel, les ressources envisageables dans le futur ${ }^{2}$. Sur ces deux axes, il est possible de distinguer quatre quadrants, chacun correspondant à une situation possible pour l'entité. La situation la plus favorable, une situation dominante comparée à celles des autres entités, correspond au quadrant 2 (les «Dominants »), avec à la fois une position forte et un potentiel important : un quadrant souvent nommé «magique » par les cabinets de conseil. Par ailleurs, si l'on considère le développement d'une entreprise au travers des différentes périodes de réorganisation

\footnotetext{
${ }^{2}$ Le potentiel d'une entreprise, souvent exprimé par le taux de croissance du chiffre d'affaires, fournit une indication sur son degré de compréhension de l'environnement, son adéquation à celui-ci. Une entreprise adaptée à son marché maitrise mieux que ses concurrents les règles du jeu. La position au sein du groupe indique les moyens dont peut disposer une entreprise. Plus sa position est forte, par exemple en termes de parts de marché ou de réseaux sociaux, plus elle engrange de revenus. Autrement dit, la position et le potentiel indiquent le niveau de ressources mobilisables immédiatement et dans le futur par l'entreprise.
} 
de son portefeuille d'activités, la trajectoire la plus favorable décrit une forme en spirale en restant proche du quadrant 2.

Figure 1 - Situation et trajectoire en spirale dans une approche matricielle

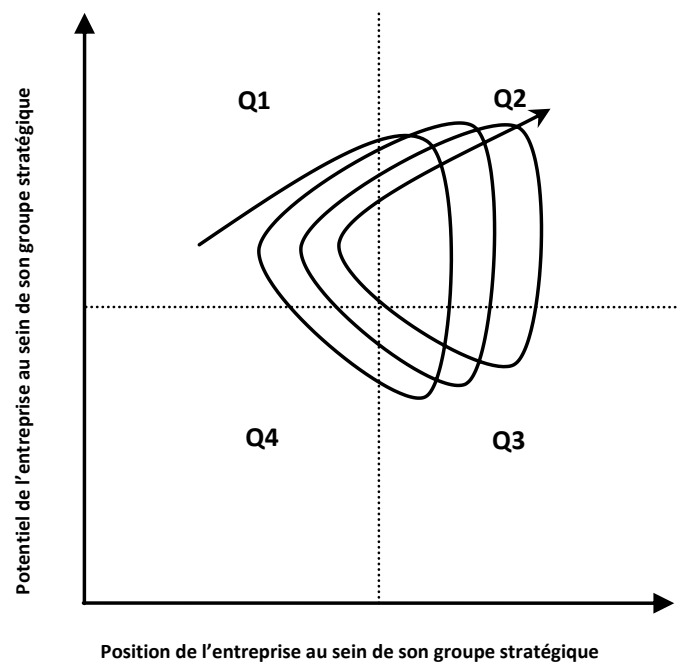

Le passage du quadrant 3 (les «Déclassés ») au quadrant 1 (les «Challengers »), c'est-à-dire d'une situation de déclin relatif à celle d'une relance à venir, est crucial. Il correspond en effet à une phase de transformation majeure où un nouveau modèle de développement doit être défini. Durant cette phase, l'entreprise doit faire face à un double enjeu : la mise en place de son futur portefeuille d'actifs en parallèle à l'abandon des activités déclinantes. En règle générale, deux phases se succèdent. La première, tournée vers l'intérieur de l'entreprise, pour faire émerger le nouveau modèle; la seconde, pour le développer. Ces deux phases correspondent à des profils d'investissement également différents. Dans la première, il est difficile de percevoir de l'extérieur la nature des changements internes en cours, à moins d'y être étroitement associé. Un investisseur «passif » ne verra que la partie déclinante des activités de l'entreprise. Dans la seconde, le nouveau modèle économique de l'entreprise est plus visible, suscitant de nouveau l'intérêt de cette dernière catégorie d'investisseurs.

Une transformation ratée, souvent trop lente, conduira l'entreprise à se diriger vers le quadrant 4 (les «Perdants »). A un certain niveau de consommation de ressources, et sans intervention 
extérieure, près de l'origine de la matrice, la disparition de l'entreprise sera inéluctable. Une transformation rapide et réussie projettera en revanche l'entreprise sur le quadrant 2.

\subsection{La matrice stratégique classique}

La matrice du cabinet Boston Consulting Group (BCG)est l'archétype de la matrice stratégique (Johnson et al., 2014). Elle permet d'évaluer l'équilibre des Domaines d'Activités Stratégiques (DAS) d'une entreprise, en positionnant ceux-ci en fonction de leur part de marché relative et du pourcentage d'accroissement de leur marché (figure 2). Ces deux paramètres vont influer sur la génération des ressources (Cash Flows) et les investissements (variation des immobilisations et du BFR).

Figure 2 - Matrice du BCG

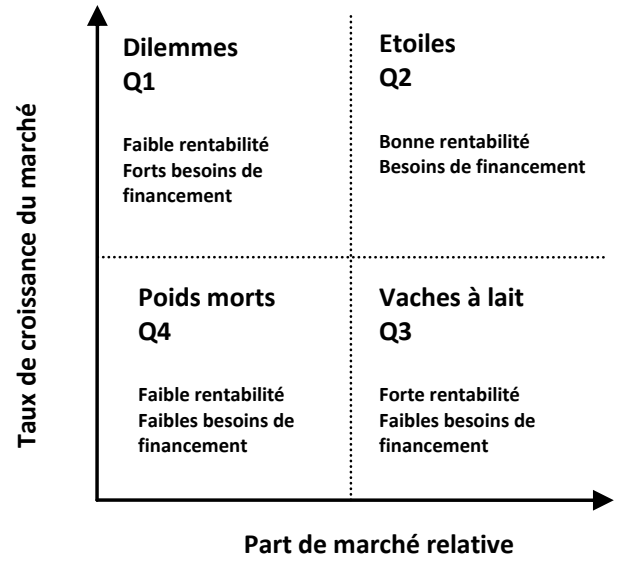

Le quadrant 2 des «étoiles » regroupe les DAS leaders sur un marché porteur. La pression concurrentielle $\mathrm{y}$ est très forte. L'entreprise doit investir lourdement pour maintenir ses positions. Ces DAS ne génèrent pas assez de cash-flows pour augmenter leur part de marché.

Le quadrant 3 des vaches à lait regroupe les DAS leaders sur un marché mature. Ces activités génèrent des profits réguliers et 
substantiels, plus de ressources qu'ils n'en consomment, ce qui permet de financer les autres DAS.

Le quadrant 1 des dilemmes regroupe les DAS en position de suiveur sur un marché en forte croissance. Ces activités présentent un risque élevé car elles nécessitent des investissements élevés, d'où justement le dilemme : faut-il continuer ou arrêter cette activité ?

Le quadrant 4 des poids morts, enfin, regroupe les DAS suiveurs sur un marché en déclin. Ces activités sont peu coûteuses, mais rapportent également peu.

A partir de cette typologie, le BCG recommande diverses stratégies. Une stratégie globale de maintien d'un portefeuille équilibré conduit à développer les étoiles, céder les dilemmes, céder également voire liquider les poids morts et «traire » les vaches à lait. Une stratégie privilégiant la rentabilité est assurée par un portefeuille ancien car les ressources générées sont importantes et les investissements minimes. En revanche, une stratégie privilégiant la prise de risques s'appuiera sur un portefeuille jeune offrant de bonnes perspectives à long terme mais des risques importants à court terme.

La matrice BCG est simple d'utilisation, nécessitant peu d'informations et permettant une lecture rapide de la stratégie d'une entreprise. En revanche, les deux dimensions retenues sont limitées pour analyser finement ces mêmes stratégies ${ }^{3}$.

De plus, même si certaines hypothèses sous-jacentes au modèle (en particulier l'autofinancement de l'entreprise) doivent être rediscutées pour tenir compte d'un contexte économique et financier nouveau, ce type de matrice reste une approche classique permettant une mise en relation cohérente des stratégies de l'entreprise avec les logiques des investisseurs (Martinet, 1990).

\footnotetext{
${ }^{3}$ La matrice Mc Kinsey repose justement sur un plus grand nombre d'informations. Les deux dimensions retenues restent toutefois l'attractivité du secteur et la position concurrentielle de l'entreprise. Les positions des DAS sont les suivantes :

- Dans les cases «Gagnants », on identifie les leaders sur un marché porteur. C'est selon Mac Kinsey la position idéale.

Dans les cases transversales, la réflexion est menée au cas par cas.

- Dans les cases « Perdants », on identifie les activités à abandonner.

Cette matrice, qui paraît plus pertinente que celle du BCG, du fait d'un plus grand nombre de données prises en compte, reste toutefois très subjective.
} 


\section{Une matrice du portefeuille international (MPI) : Principe et illustration}

L'impact de l'internationalisation sur la performance en regard de sa dynamique reste l'objet d'un débat académique, comme nous l'avons souligné plus haut. Malgré et à cause de cela, l'activité internationale d'une entreprise est particulièrement suivie par les investisseurs, pour au moins trois grandes raisons. Tout d'abord, l'internationalisation d'une entreprise montre sa capacité à élargir sa base de clientèle au-delà de son territoire domestique. Ensuite, une synergie et une cohérence doivent être trouvées entre les différents marchés exploités. Enfin, c'est le moyen naturel pour une entreprise d'assurer sa croissance à long terme.

Les deux premières raisons reposent sur des arguments essentiellement qualitatifs. La question de la croissance est en revanche suivie avec des indicateurs quantitatifs. C'est l'objet principal de la matrice. Pour autant, la position des entreprises sur cette dernière s'explique justement par des raisons qualitatives.

\subsection{Principes de construction}

Nous nous intéressons dans cet articleà la gestion du portefeuille d'activités à l'international des entreprises, dans une approche matricielle telle qu'elle a été développée plus haut. Par souci de simplification, nous nommerons cette matrice «Matrice du Portefeuille International » (MPI).

Les principes de construction reposent fondamentalement sur la définition des deux axes de la matrice. Nous raisonnons sur le portefeuille d'activités del'entreprise et distinguons donc les différents marchés géographiques de l'entreprise. Ce choix, évidemment discutable, conduit cependant à établir une corrélation positive, assez naturelle (on établit en général des prévisions de croissance d'activité en fonction des taux de croissance économique), entre les activités de l'entreprise et la croissance économique ${ }^{4}$. De fait, l'indicateur utilisé pour l'axe des abscisses découle de ce premier choix : la part de marché à l'international.

\footnotetext{
${ }^{4}$ Cette hypothèse apparaît en fait peu contraignante, car le raisonnement peu s'accommoder d'une relation négative entre l'activité de l'entreprise et le cycle économique. Nous préférons cependant exposer la matrice pour une activité a priori pro-cyclique.
} 
Par ailleurs, l'axe du taux de croissance des marchés doit tenir compte d'une présence de l'entreprise sur plusieurs zones géographiques.Cet indicateur doit donc représenter une moyenne pondérée du taux de croissance des marchés à l'international. Pour rester cohérent avec l'indicateur précédent, on utilisera le taux de croissance du PIB comme base de calcul. Par ailleurs, la recherche d'un relais de croissance étant ici l'argument majeur d'une stratégie d'internationalisation, il paraît nécessaire de raisonner à partir du gain de croissance procuré par les marchés étrangers par rapport au marché domestique.

$\mathrm{Au}$ final, le portefeuille d'activités à l'international de l'entreprise pourra se situer dans l'un des quatre quadrants de la MPI (figure 3), avec une différence notable par rapport à ceux présentés plus haut avec les matrices stratégiques. Ainsi, les quadrants 2 et 4 représentent ici les positions clés de l'entreprise. En effet, le quadrant 2 regroupe les entreprises avec une part de marché à l'international importante, sur des zones en croissance plus forte que le marché domestique. Nous le nommerons le quadrant des «Dragons». Le quadrant 4, à l'opposé, distingue les entreprises faiblement internationalisées, mais présentes sur des marchés apportant peu de gains de croissance. Nous utiliserons pour ce quadrant le terme d' «Ours». Si l'on se réfère à ce dernier argument, ces deux positionnements devraient néanmoins influer positivement sur la performance et apporter des satisfactions aux investisseurs.

En revanche, les quadrants 1 et 3 reflètent des positions plus riches à interpréter. Le quadrant 1 regroupe en effet les entreprises faiblement internationalisées, mais sur des marchés plus dynamiques que le marché domestique (quadrant 1 - Dilemme). Aussi, soit les entreprises s'y rattachant n'exploitent pas un potentiel de croissance à l'international (argument négatif), soit au contraire elles conduisent une internationalisation progressive avisée (argument positif). Dans les deux cas, il faut recourir à une analyse plus fine de la trajectoire de ces entreprises, les «Renards». A l'inverse, le quadrant 3 reflète bien une situation de déclin ou de danger pour l'entreprise, encore fortement présente à l'international sur des marchés moins dynamiques que le marché domestique: soit l'entreprise subit le déclin des marchés où elle est installée, soit elle cherche à être présente fortement à l'étranger sans se préoccuper de l'attractivité des marchés non domestiques. Nous les appellerons les «Mammouths». 
Figure 3 : Matrice du portefeuille international (MPI)

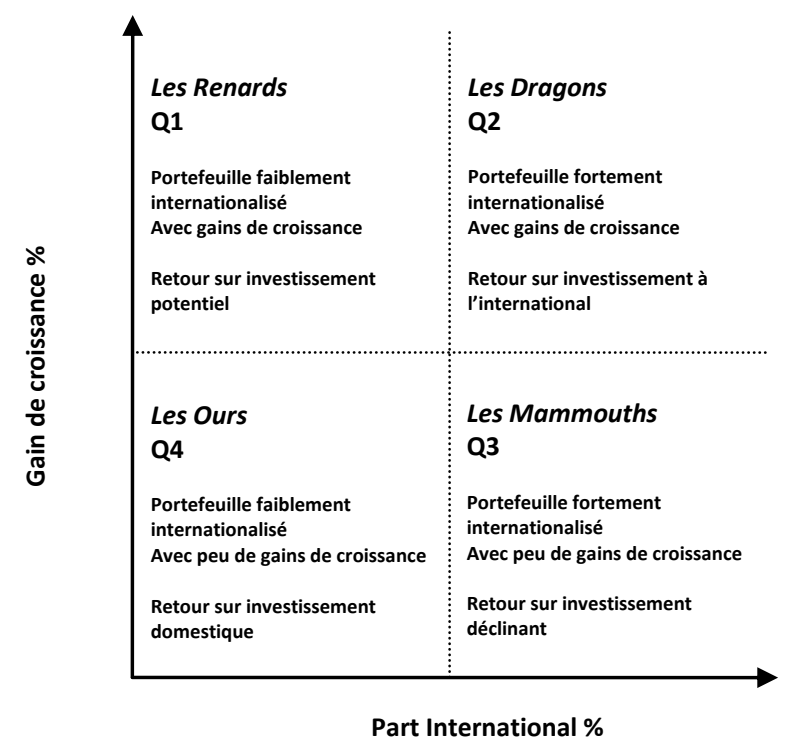

\subsection{Une application au secteur des composants et équipements électriques}

Pour illustrer la MPI, nous avons retenu un échantillon de sociétés du secteur des équipements et composants électriques. Ce choix s'explique par trois raisons principales importantes. Tout d'abord, ce marché est dominé par des groupes globaux, présents sur toutes les lignes de produits, permettant l'hypothèse forcée d'un rattachement au même groupe stratégique, ce qui légitime évidemment une approche comparative ${ }^{5}$. Par ailleurs, les grandes entreprises sont mieux intégrées dans l'économie mondiale (Besanko et al., 2004). Ensuite, le marché des composants et équipements électriques, estimé à environ 114 milliards de $€$ en 2013, a connu une croissance annuelle cumulée de 7,6\% entre 2009 et 2013, les ventes à l'international expliquant l'essentiel de la croissance des entreprises ${ }^{6}$. Enfin, le secteur, historiquement peu concentré (pour 2013, on obtient

\footnotetext{
${ }^{5}$ Les biais liés à des portefeuilles d'activités non parfaitement substituables doivent être gardés à l'esprit.

${ }^{6}$ Source : Etude Marketresearch (2014).
} 
un index d'Herfindhal inférieur à 1600), connait des opérations de rapprochement entre acteurs (e.g. l'acquisition en cours de l'activité énergie d'Alstom par General Electric), en rapport avec la question stratégique de l'énergie ${ }^{7}$.

En se fondant sur les principes précédents se rapportant à l'identification d'un groupe stratégique, nous avons pu retenir pour cette étude 13 sociétés cotées (tableau 1), cette dernière caractéristique étant nécessaire pour étudier l'influence du positionnement à l'international sur la performance financière.

Tableau 1: Description des sociétés retenues dans l'échantillon

\begin{tabular}{|c|c|c|c|c|c|c|c|}
\hline & & \multicolumn{2}{|c|}{$\begin{array}{c}\text { Chiffre } \\
\text { d'affaires }^{1}\end{array}$} & \multicolumn{2}{|c|}{$\begin{array}{c}\text { Part export } \\
(\%)\end{array}$} & \multicolumn{2}{|c|}{ ROE (\%) } \\
\hline Société & Pays & 2009 & 2013 & 2009 & 2013 & 2009 & 2013 \\
\hline $\mathrm{ABB}$ & Sweden & 31.8 & 41.8 & 97,99 & 97,99 & 21,04 & 14,92 \\
\hline Alstom & France & 19.6 & 29.7 & 89,91 & 93,21 & 29,38 & 11,08 \\
\hline Emerson electric & USA & 20.1 & 24.6 & 56,79 & 59,46 & 20,15 & 19,28 \\
\hline General electric & USA & 154.4 & 146 & 50,91 & 53,01 & 8,99 & 9,76 \\
\hline Honeywell & USA & 29.9 & 39 & 39,90 & 41,16 & 24,45 & 22,54 \\
\hline Johnson Controls & USA & 28.4 & 42.7 & 61,05 & 60,96 & $-3,68$ & 9,57 \\
\hline Legrand & France & 3.5 & 4.4 & 71,52 & 76,37 & 12,16 & 16,41 \\
\hline Melrose & UK & 1.2 & 1.7 & 84,46 & 87,68 & 10,39 & 25,81 \\
\hline Nidec & Japan & 586 & 875.1 & 74,23 & 81,85 & 15,27 & 10,89 \\
\hline Regal-Beloit & Canada & 1.8 & 3 & 26,01 & 34,82 & 8,13 & 5,84 \\
\hline Schneider & France & 15.7 & 23.5 & 90,32 & 92,78 & 7,52 & 11,32 \\
\hline Siemens & Germany & 76.5 & 75.8 & 84,96 & 85,83 & 9,15 & 15,40 \\
\hline United tech & USA & 52.4 & 62.6 & 46,61 & 42,52 & 19,54 & 17,27 \\
\hline
\end{tabular}

Les données concernant l'activité des entreprises sont tirées de la base Datastream et des documents de référence des sociétés, en particulier pour la ventilation du chiffre d'affaires par zones géographiques $^{8}$. Les données économiques (PIB) proviennent de la Banque mondiale.

Nous retenons 5 années suivant la crise des subprimes (20092013) comme période de référence, d'une part pour disposer d'un

\footnotetext{
${ }^{7}$ Par ailleurs, l'activité du secteur est de nature pro-cyclique, ce qui permet une lecture directe de la matrice.

${ }^{8}$ Le recours à ces données secondaires des entreprises contraint sur le choix des zones géographiques et conduit ainsi à des regroupements de pays dont l'homogénéité en termes de croissance est questionnable. D'un autre côté, le découpage géographique opéré par les entreprises retenues repose quasiment sur les mêmes bases, ce qui atténue en partie le biais précédent.
} 
contexte économique commun aux entreprises retenues (toutes font partie de l'aire économique «occidentale »), d'autre part car la crise a rendu la question de l'activité à l'international plus cruciale. Par ailleurs, le recours à 5 années permet d'effectuer une analyse statistique sur un panel de 65 données (13 sociétés par 5 années).

La matrice du portefeuille international (MPI) que nous proposons permet ainsid'étudier l'impact du positionnement des entreprises à l'international sur leur performance financière. En ce qui concerne le premier point, nous croisons l'axe horizontal de la part du chiffre d'affaires à l'international avec un axe vertical, qui mesure le gain de croissance tiré des activités à l'international ${ }^{9}$. Ceci nous permet d'identifier4 quadrants reflétant des positionnements caractéristiques.Comme cela a été exposé plus haut, l'approche en termes de gains de croissance conduit à opposer deux positionnements de base et permet d'analyser des situations intermédiaires. Ainsi, comme le montrent la figure 4 ci-après, une entreprise peut privilégier son marché domestique, si celui-ci lui apporte une croissance supérieure aux marchés extérieurs. Dans ce cas, sa part de marché à l'international sera plus faible que la moyenne. On retrouve dans cette situation l'essentiel des sociétés américaines sur la période. A l'inverse, si les gains de croissance à l'international sont conséquents, la part de marché à l'international devrait également être élevée par rapport à la moyenne. C'est la situation d'une manière générale des sociétés européennes. Les deux autres quadrants illustrent des positionnements a priori moins cohérents quant à l'activité internationale. En effet, soit l'entreprise choisit d'être très présente à l'international, mais sur des marchés peu dynamiques (ABB par exemple), soit elle limite son exposition à l'international, sur des marchés pourtant porteurs (le cas à la marge d'Emerson).

La régression de la variable «gains de croissance » effectuée sur la variable «part à l'international » sur l'ensemble du panel donne un résultat significatif, ce qui confirme les deux positionnements de base identifiés.

\footnotetext{
${ }^{9}$ Nous recalculons le pourcentage à l'export en tenant compte du poids du PIB domestique dans le PIB mondial.La croissance moyenne des marchés à l'international de l'entreprise est rapportée à celle du marché domestique, ce qui permet de raisonner en termes de gains de croissance.
} 
Figure 4 : MPI en 2009 - Secteur des équipements électriques

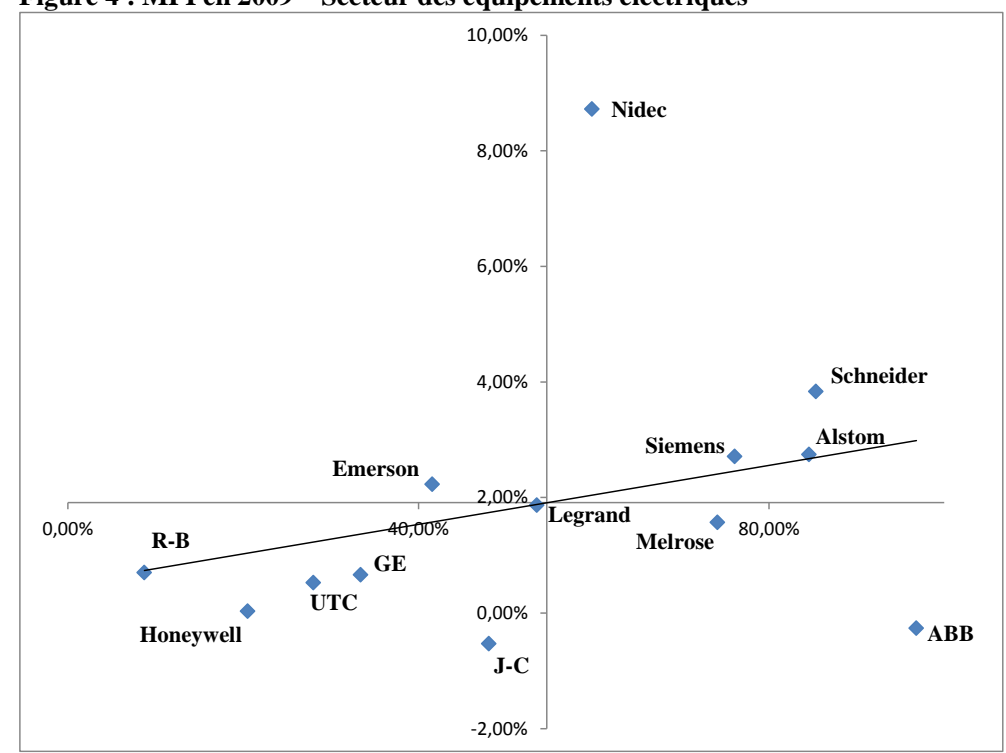

Pour confirmer le lien entre le positionnement dans l'un des quadrants de base 2 et 4 et la performance financière, nous classons pour chaque année les entreprises en deux catégories, celles positionnées dans les quadrants 2 et 4 et celles positionnées dans les deux autres quadrants. Nous étudions ensuite la relation statistique entre cette nouvelle position modifiée et un indicateur de performance financière classique (ROE) avec les données du panel. Le résultat est positif et significatif, ce qui confirme l'influence d'un des deux positionnements de base sur la performance des entreprises (tableau 2).

Tableau 2 : Résultats de la régression

\begin{tabular}{l|llll} 
ROE & Coef. & Std. Err. & T & P $>|t|$ \\
Q2-Q4 & 0.046191 & 0.019027 & 2.42 & 0.018 \\
N = 65, F = 5,8931, Prob $>\mathrm{F}=0.018 ; \mathrm{R}^{2}=0.2925$
\end{tabular}

Comme conséquence de ce résultat, il est également possible d'en inférer l'origine majeure de la sous-performance d'entreprises situées sur les quadrants de base 2 et 4: une organisationinsuffisamment efficace des activités «domestiques » pour les entreprises du quadrant 4 , une organisation insuffisamment 
efficace des activités à l'international pour les entreprises du quadrant 2.

On peut par ailleurs illustrer la situation des entreprises positionnées sur l'un des deux autres quadrants par le cas d'ABB (tableau 3). Avec une part à l'international particulièrement conséquente sur toute la période, mais des gains de croissance faibles, la rentabilité financière diminue significativement sur la période ${ }^{10}$.

Tableau 3 : Le cas de la société $\mathrm{ABB}$ de 2009 à 2013

\begin{tabular}{|l|r|r|r|r|l|}
\hline & \multicolumn{1}{|c|}{2013} & \multicolumn{1}{|c|}{2012} & \multicolumn{1}{c|}{2011} & \multicolumn{1}{c|}{2010} & \multicolumn{1}{c|}{2009} \\
\hline Part à l'international & $97,99 \%$ & $97,99 \%$ & $97,99 \%$ & $97,99 \%$ & $97,99 \%$ \\
\hline Gains de croissance & $0,10 \%$ & $1,26 \%$ & $1,10 \%$ & $0,98 \%$ & $-0,26 \%$ \\
\hline ROE & $14,92 \%$ & $15,99 \%$ & $20,08 \%$ & $17,21 \%$ & $21,04 \%$ \\
\hline
\end{tabular}

\section{Conclusion et limites de l'étude}

La valorisation du portefeuille international des entreprises est devenue fondamentale avec la globalisation économique. La matrice présentée dans cet article fournit une aide indispensable aux investisseurs pour estimer celui-ci. Pour autant, quelques précisions et précautions dans l'établissement et l'interprétation de la matrice doivent être rappelées.

On gagnera tout d'abord à mener l'analyse par domaine d'activité. L'approche retenue ici suppose un portefeuille d'activités comparable entre entreprises, donc le recours à une logique de type groupe stratégique, dont les facteurs de regroupement sont questionnables sur le moment et dans le temps. Par ailleurs, la zone géographique retenue (groupe de pays, pays, région...) pour le calcul du taux de croissance économique doit être cohérente avec le niveau d'analyse de l'activité de l'entreprise, et donc de sa taille. Sur ce point, la qualité d'analyse sera systématiquement supérieure si les données utilisées permettent un calcul de la croissance par pays. Enfin, pour tenir compte de la logique anticipative des investisseurs financiers, il pourrait être utile, d'une part de calculer la croissance économique en termes de potentiel, d'autre part d'introduire un décalage entre la position des entreprises sur la matrice et la réaction des investisseurs.

\footnotetext{
${ }^{10}$ Même si l'impact du coût d'entrée de nouveaux marchés ne peut être complètement écarté.
} 
Plusieurs paramètres influant sur la performance financière des entreprises devraient également être pris en considération ${ }^{11}$. Tout d'abord, compte tenu de l'importance de la fiscalité, tant au niveau de la maison-mère d'un groupe que de ses filiales à l'international (Errunza et Senbet, 1981; Leibfritz et al., 1997), cette variable nécessiterait une analyse approfondie. Par exemple, le taux d'imposition au Japon est particulièrement faible. De plus, l'impact des taux d'intérêt auxquels se financent les entreprises n'est pas considéré dans notre étude. On sait ainsi que le Japon procure des taux structurellement faibles à ces entreprises, mais comment en particulier la politique très accommodante de la banque centrale américaine depuis la crise a-t-elle exercée ses effets ? Enfin, le raisonnement proposé s'appuie sur la notion de croissance et pas de marge. Or, le degré de concentration de chaque marché peut également influer sur les choix d'activité à l'international.

La notion de «marché domestique », en particulier pour des multinationales aux chaînes de valeur intégrées mondialement, peut également interpelée. Elle suppose l'existence d'un paramètre justifiant une approche économique différenciée entre marché « domestique » et marché « étranger», comme par exemple la fiscalité ou le pouvoir politique. En écho au commentaire précédent, ce point doit certainement être approfondi. Dans cette perspective, une application de l'approche matricielle à des PME, même avec l'existence d'autres limites liées en particulier au degré d'internationalisation, apporterait quelques clarifications.

Ces différentes remarques au final pour nous rappeler que la grille d'analyse proposée doit servir d'aide à la prise de décision, sans pouvoir remplacer une étude poussée nécessaire à la compréhension d'un phénomène nécessairement complexe.

\footnotetext{
11 Ainsi, le $\mathrm{R}^{2}$ d'environ $30 \%$ trouvé sur la relation entre positionnement à l'international et performance financière, quoique non négligeable, suggère d'autres facteurs explicatifs.
} 


\section{Références}

ANNAVARJULA M., BELDONA S., «Multinationality-performance relationship: A review and reconceptualization », The International Journal of Organization Analysis, tome VIII, N¹, 2000, p48.-67.

CONTRACTOR F. J., «Is international business good for companies? The evolutionary or multi-stage theory of internationalization vs. the transaction cost perspective », Management International Review, tome XLVII, $\mathrm{N}^{\circ}$ 3, juin, 2007, p463.-475.

Gomez-Mejia L. R., PALICH L.E., «Cultural diversity and the performance of multinational firms », Journal of International Business Studies, tome XXVIII, N², 1997, p309.-335.

ELANGO B., SETHI S. P., «An exploration of the relationship between country of origin (COE) and the internationalization-performance paradigm », Management International Review, tome XLVII, $\mathrm{N}^{\circ} 3$, 2007, p.369.-392.

ERRUNZA V. R., SENBET L.W., «The effects of international operations on the market value of the firm: Theory and evidence »,The Journal of Finance, tome XXXVI, N², mai, 1981, p401.-417.

JOHANSON J., VAHLNE J.E., «The Mechanism of Internationalisation », International Marketing Review, tome VII, $\mathrm{N}^{\circ} 4,1990$, p11.-24.

Hitt M. A., Tihanyi L., Miller T., Connelly B., «International diversification: Antecedents, outcomes, and moderators », Journal of Management, tome XXXII, $\mathrm{N}^{\circ} 6,2006$, p831.-867.

HSU C. C., BoGGS D. J., «Internationalization and performance: Traditional measures and their decomposition », Multinational Business Review, tome XI, $\mathrm{N}^{\circ} 3,2003$, p23.-49.

Johnson G., Whittington R., Scholes K., Angwin D., Regner P.,FrÉRY F., Stratégique (10e édition), Pearson, Paris, 2014.

LEIBFrITZ W., ThORNTON J., BIBBEE A., Taxation and economic performance, OECD, 1997.

LU J. W., BEAMISH P. W., «International diversification and firm performance: The S-curve hypothesis », Academy of management journal, tome XLVII, N4, 2004, p598.-609.

MARTinet A.-C., Diagnostic stratégique, Vuibert, Paris, 1990.

MiCHEL A., SHAKED I., "Multinational corporations vs. domestic corporations: Financial performance and characteristics », Journal of International Business Studies, tome XVII, N³, 1986, p89.-100.

PRANGE C., VERDIER S., «Dynamic capabilities, internationalization processes and performance », Journal of World Business, tome XLVI, $\mathrm{N}^{\circ} 1,2011, \mathrm{p} 126 .-133$. 
QIAN G., LI L., QIAN,Z., «Regional diversification and firm performance », Journal of International Business, tome XXXIX, N², 2008, p197.-214.

REID S., "Firm internationalization, transaction costs and strategic choice », International marketing review, tome 1, №2, hiver, 1983, p43.-61.

RialP A., RialP J., KNIGHT G.A., "The phenomenon of early internationalizing firms: what do we know after a decade (1993-2003) of scientific inquiry? »International Business Review, tome XIV, avril, 2005, p147.-166.

RIAHI-BELKAOUI A., "The effects of the degree of internationalization on firm performance », International Business Review, tome VII, N³, 1998, p315.-321.

RUIGROK W., WAGNER H., «Internationalization and firm performance: Meta-analytic review and future research directions », annual meeting of the Academy of International Management, Stokholm, Suède, 2004.

ThOMAs D. E., EDEN E., «What is the shape of the multinationalityperformance relationship? »,Multinational Business Review, tome XII, $\mathrm{N}^{\circ} 1,2004, \mathrm{p} 89 .-110$.

TSAO S. M., CHEN G.Z., «The impact of internationalization on performance and innovation: The moderating effects of ownership concentration », Asia Pacific Journal of Management, tome XXIX, $\mathrm{N}^{\circ} 10,2012$, p617.-642.

Weerawardena J., Mort G. S., Liesch P. W., « Conceptualizing accelerated internationalization in the born global firm: A dynamic capabilities perspective », Journal of World Business, tome XLII, $\mathrm{N}^{\circ} 3$, 2007, p294.-306. 